\title{
Spatially resolved quantum plasmon modes in metallic nano-films from first-principles
}

\author{
Andersen, Kirsten; Jacobsen, Karsten W.; Thygesen, Kristian S.
}

Published in:

Physical Review B Condensed Matter

Publication date:

2012

Document Version

Publisher's PDF, also known as Version of record

Link back to DTU Orbit

Citation $(A P A)$ :

Andersen, K., Jacobsen, K. W., \& Thygesen, K. S. (2012). Spatially resolved quantum plasmon modes in metallic nano-films from first-principles. Physical Review B Condensed Matter, 86(24), 245129.

\section{General rights}

Copyright and moral rights for the publications made accessible in the public portal are retained by the authors and/or other copyright owners and it is a condition of accessing publications that users recognise and abide by the legal requirements associated with these rights.

- Users may download and print one copy of any publication from the public portal for the purpose of private study or research.

- You may not further distribute the material or use it for any profit-making activity or commercial gain

- You may freely distribute the URL identifying the publication in the public portal

If you believe that this document breaches copyright please contact us providing details, and we will remove access to the work immediately and investigate your claim. 


\title{
Spatially resolved quantum plasmon modes in metallic nano-films from first-principles
}

\author{
Kirsten Andersen, ${ }^{1}$ Karsten W. Jacobsen, ${ }^{1}$ and Kristian S. Thygesen ${ }^{1,2, *}$ \\ ${ }^{1}$ Center for Atomic-scale Materials Design (CAMD), Department of Physics, Technical University of Denmark, \\ DK-2800 Kgs. Lyngby, Denmark \\ ${ }^{2}$ Center for Nanostructured Graphene (CNG), Department of Physics, Technical University of Denmark, DK-2800 Kgs. Lyngby, Denmark
}

(Received 14 August 2012; published 26 December 2012)

\begin{abstract}
Electron energy loss spectroscopy (EELS) can be used to probe plasmon excitations in nanostructured materials with atomic-scale spatial resolution. For structures smaller than a few nanometers, quantum effects are expected to be important, limiting the validity of widely used semiclassical response models. Here we present a method to identify and compute spatially resolved plasmon modes from first-principles based on a spectral analysis of the dynamical dielectric function. As an example we calculate the plasmon modes of 0.5 to $4 \mathrm{~nm}$ thick Na films and find that they can be classified as (conventional) surface modes, subsurface modes, and a discrete set of bulk modes resembling standing waves across the film. We find clear effects of both quantum confinement and nonlocal response. The quantum plasmon modes provide an intuitive picture of collective excitations of confined electron systems and offer a clear interpretation of spatially resolved EELS spectra.
\end{abstract}

DOI: 10.1103/PhysRevB.86.245129

PACS number(s): 73.21.-b, 71.45.Gm, 73.22.Lp, 78.20.-e

\section{INTRODUCTION}

The dielectric properties of a material are to a large extent governed by the collective eigenmodes of its electron system known as plasmons. ${ }^{1}$ Advances in spectroscopy and materials preparation have recently made it possible to study and control light-matter interactions at the nanometer length scale where particularly the surface plasmons play a key role. ${ }^{2,3}$ While the ultimate goal of nanoplasmonics as a platform for ultrafast and compact information processing remains a challenge for the future, the unique plasmonic properties of metallic nanostructures have already been utilized in a number of applications including molecular sensors, ${ }^{4,5}$ photocatalysis, ${ }^{6}$ and thin-film solar cells. ${ }^{7}$

Electron energy loss spectroscopy (EELS) has been widely used to probe plasmon excitations in bulk materials and their surfaces. More recently, the use of highly confined electron beams available in transmission electron microscopes has made it possible to measure the loss spectrum of lowdimensional structures on a subnanometer length scale and with $<0.1 \mathrm{eV}$ energy resolution. ${ }^{8}$ Because the loss spectrum is dominated by the plasmons this technique provides a means for visualizing the real-space structure plasmon excitations. ${ }^{9}$

All information about the plasmons of a given material is contained in its frequency-dependent dielectric function $\epsilon$, which relates the total potential in the material to the externally applied potential to linear order,

$$
\phi_{\mathrm{ext}}(r, \omega)=\int \epsilon\left(r, r^{\prime}, \omega\right) \phi_{\mathrm{tot}}\left(r^{\prime}, \omega\right) d r^{\prime} .
$$

(We have specialized to the case of longitudinal fields which can be represented by scalar potentials.) In the widely used Drude model, one neglects the spatial variation of $\epsilon$ and describes the frequency dependence by a single parameter, the bulk plasmon frequency $\omega_{p}=\sqrt{n e^{2} / m \epsilon_{0}}$, where $n$ is the average electron density of the material. ${ }^{2}$ For metal surfaces the Drude model predicts the existence of surface plasmons with frequency $\omega_{s}=\omega_{p} / \sqrt{2}$. While the Drude model provides a reasonable description of plasmons in simple metal structures in the mesoscopic size regime, it fails to account for the dispersion (wave vector dependence) of the plasmon energy in extended systems and predicts a divergent field enhancement at sharp edges. These unphysical results are to some extent remedied by the semiclassical hydrodynamic models which can account for spatial nonlocality of $\epsilon$ and smooth charge density profiles at the metal-vacuum interface. ${ }^{2,10,11}$ Still, for materials other than the simple metals and for truly nanometer sized structures, predictive modeling of plasmonic properties must be based on a full quantum mechanical description. ${ }^{12,13}$ The calculation of plasmon energies and EELS spectra of periodic solids from first-principles is a well established discipline of computational condensed matter physics. ${ }^{14-17}$ Here we apply these powerful methods to systematically map out the real space structure of plasmon excitations at the nanometer length scale.

In this paper we present a general method to calculate spatially resolved plasmon modes from first-principles. We apply the method to $\mathrm{Na}$ films of a few nanometer thickness where effects of quantum confinement and nonlocal response are expected to be important. The plasmon modes we find can be categorized as surface modes located mainly outside the metal surface, subsurface modes located just below the surface, and bulk modes. Very intuitively, the bulk plasmon modes resemble standing waves with nodes at the film surfaces. However, only plasmons with oscillation periods larger than $10 \AA$ are found as a result of Landau damping which suppresses the strength of plasmon modes with smaller oscillation periods. Finally, we calculate the spatially resolved EELS spectrum of the metal films and show that all its features can be traced to excitation of specific plasmon modes.

\section{METHOD}

According to Eq. (1), a self-sustained charge density oscillation $\rho(r, \omega)$ can exist in a material if the related potential, satisfying the Poisson equation $\nabla^{2} \phi=-4 \pi \rho$ (atomic units are used throughout), obeys the equation

$$
\int \epsilon\left(r, r^{\prime}, \omega\right) \phi\left(r^{\prime}, \omega\right) d r^{\prime}=0 .
$$


In general, this equation cannot be exactly satisfied because the dielectric function will have a finite imaginary part originating from single-particle transitions which will lead to damping of the charge oscillation. ${ }^{18}$ We therefore require only that the real part of $\epsilon$ vanishes and use the following defining equation for the potential associated with a plasmon mode,

$$
\int \epsilon\left(r, r^{\prime}, \omega_{n}\right) \phi_{n}\left(r^{\prime}, \omega_{n}\right) d r^{\prime}=i \Gamma_{n} \phi_{n}\left(r, \omega_{n}\right),
$$

where $\Gamma_{n}$ is a real number. Mathematically, the plasmon modes are thus the eigenfunctions corresponding to purely imaginary eigenvalues of the dielectric function. Physically, they represent the potential associated with self-sustained charge-density oscillations damped by electron-hole pair formations at the rate $\Gamma_{n}$.

It is instructive to consider the dielectric function in its spectral representation

$$
\epsilon\left(r, r^{\prime}, \omega\right)=\sum_{n} \epsilon_{n}(\omega) \phi_{n}(r, \omega) \rho_{n}\left(r^{\prime}, \omega\right),
$$

where the left and right eigenfunctions satisfy the usual orthonormality relation

$$
\left\langle\phi_{n}(\omega) \mid \rho_{m}(\omega)\right\rangle=\delta_{n m} .
$$

In the Appendix we show that the left and right eigenfunctions are related through Poisson's equation

$$
\nabla^{2} \phi_{n}(r, \omega)=-4 \pi \rho_{n}(r, \omega),
$$

and thus correspond to the potential and charge density of the dielectric eigenmode, respectively. Physically, the condition (5) expresses the fact that the different dielectric eigenmodes for a given frequency $\omega$ are electrodynamically decoupled. The inverse dielectric function $\epsilon^{-1}\left(r, r^{\prime}, \omega\right)$ is obtained by replacing the eigenvalues $\epsilon_{n}(\omega)$ in Eq. (4) by $1 / \epsilon_{n}(\omega)$.

When the imaginary part of the eigenvalue $\epsilon_{n}(\omega)$ does not vary too much around the plasmon frequency $\omega_{n}$, the condition (3) is equivalent to the condition that

$$
\operatorname{Im} \epsilon_{n}\left(\omega_{n}\right)^{-1} \text { is a local maximum. }
$$

This is the case for most of the plasmon modes of the simple metal films studied in this work. However, for the high energy bulk modes we found that the variation in $\operatorname{Im} \epsilon_{n}(\omega)$ can shift the local maximum of $\operatorname{Im} \epsilon_{n}(\omega)^{-1}$ away from the point where $\operatorname{Re} \epsilon_{n}(\omega)=0$. (The effect can be even stronger for more complex materials with interband transitions. ${ }^{19}$ ) In such cases the condition (7) rather than (3) should be used to define the plasmon energy. Importantly, however, the eigenfunctions $\phi_{n}(r, \omega)$ do not change significantly when $\omega$ is varied between the frequencies given by the two criteria, and thus the spatial form of the plasmon remains well defined.

In the case of metallic films where the in-plane variation of $\epsilon$ and $\phi_{n}$ can be assumed to have the forms $\exp \left(i q_{\|} \cdot\left(r_{\|}-r_{\|}^{\prime}\right)\right)$ and $\exp \left(i q_{\|} \cdot r_{\|}\right)$, respectively, Eq. (3) can be written

$$
\int \epsilon\left(q_{\|}, z, z^{\prime}, \omega_{n}\right) \phi_{n}\left(z^{\prime}, \omega_{n}\right) d z^{\prime}=i \Gamma_{n} \phi_{n}\left(z, \omega_{n}\right) .
$$

We stress that despite the atomic variation in the potential we have found that local field effects are negligible within the plane justifying this assumption.
All the calculations were performed with the electronic structure code GPAW $^{20,21}$ using the Atomic Simulation Environment. $^{22}$ The Na films were modeled in a supercell with periodic boundary conditions imposed in all directions. The minimal Na unit cell was used in the plane of the film, and $30 \AA$ of vacuum was included in the direction perpendicular to the film to separate the periodic images. The single-particle wave functions and energies were computed on a real-space grid with a grid spacing $0.18 \AA$, and the Brillouin zone was sampled by $64 \times 64 k$ points. The local density approximation (LDA) functional was used for exchange and correlation. The noninteracting density response function $\chi^{0}$ was calculated from the single-particle states of a density functional theory (DFT) calculation using a $50 \mathrm{eV}$ energy cutoff for the plane wave basis and including states up to $15 \mathrm{eV}$ above the Fermi level. The frequency dependence of the dielectric function was sampled on the real frequency axis using a grid from 0 to $20 \mathrm{eV}$ with a $0.1 \mathrm{eV}$ spacing. We checked that our results were converged with respect to all the parameters of the calculation. The microscopic dielectric function was calculated from $\chi^{0}$ using the random phase approximation (RPA). We note in passing that this DFT-RPA level of theory yields highly accurate bulk and surface plasmon energies (within a few tenths of an electron volt) of the simple metals. ${ }^{17}$

To obtain the plasmon modes, the dielectric function is diagonalized in a plane-wave basis on each point of a uniform frequency grid. To simulate an isolated film, the obtained eigenmode potentials were corrected to remove the effect of interactions with films in the other supercells, i.e., the periodic boundary conditions from the DFT calculation (this is most important for small $q_{\|}$vectors). In general the eigenmodes were found to be complex valued, reflecting the spatial variation in the phase of the plasmon potential. This phase variation is, however, rather weak for the present system, and by a suitable choice of the overall phase the modes can be made almost real (>90\%). For this reason only the real part of the plasmon modes are shown in this work.

\section{RESULTS}

In the left panel of Fig. 1 we show the plasmon modes $\phi_{n}(z)$ (red) and corresponding charge densities $\rho_{n}(z)$ (blue) obtained for a 10 atom thick $\mathrm{Na}$ film terminated by (100) surfaces. Figure 2 shows the real part of the eigenvalues of $\epsilon$ for the ten layer film. The red dots indicate the plasmon frequencies $\omega_{n}$, where the real part of an eigenvalue crosses the real axis from below. Note that the point where an eigenvalue crosses the real axis from above corresponds roughly to the energy of the individual single-particle transitions contributing to the plasmon state. Thus the distance between the two crossing points, $\tilde{\alpha}$, represents the Coulombic restoring force of the plasma oscillation. The eigenfunctions of $\epsilon$ corresponding to the indicated eigenvalues are the plasmon modes shown in Fig. 1. The energies and strengths $\alpha_{n}$ of the plasmon modes are listed to the left. The strength is determined by fitting a single-pole model

$$
\epsilon_{n, 1 p}(\omega)=1-\frac{\alpha_{n}}{\omega-\omega_{n, 0}+i \gamma_{n}}
$$

to the value and slope of the relevant eigenvalue branch of $\epsilon$ at the point $\omega=\omega_{n}$; see Fig. 2 . 

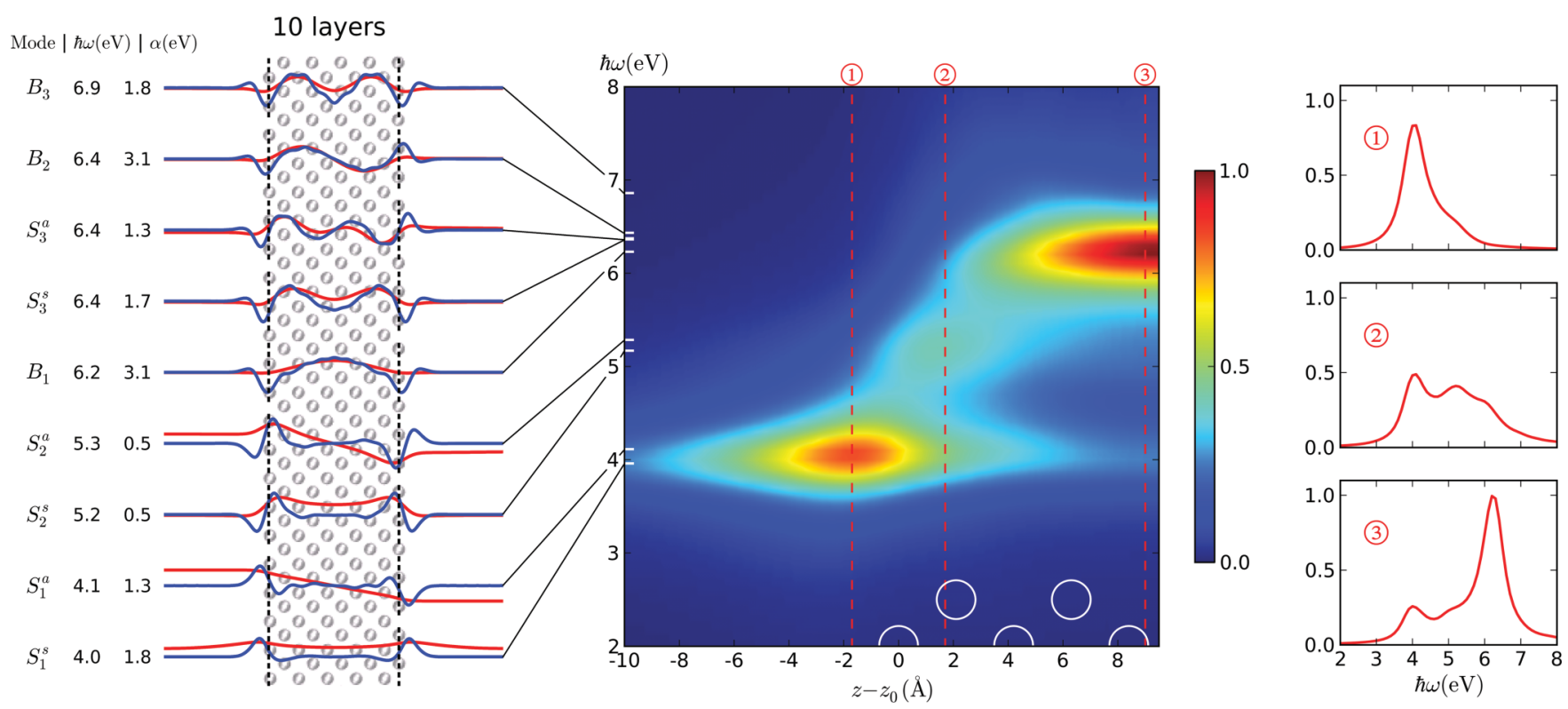

FIG. 1. (Color online) (Left) Spatial profile of the plasmon modes in the direction normal to the film for in-plane momentum transfer $q_{\|}=0.1 \AA^{-1}$. The red (blue) curves show the potential (density) associated with the plasmon excitation. Mode type, energy, and strength $\alpha$ are shown to the left. (Middle) Contour plot of the energy loss of an in-plane electron beam as it is scanned across the film. Only the left half of the film is shown, and the positions of the $\mathrm{Na}$ atoms are indicated by white circles at the bottom. The three bright features in the spectrum originate from energy loss due to excitation of three types of plasmons, namely, the surface plasmon mode $S_{1}$, the subsurface mode $S_{2}$, and the lowest bulk plasmon modes $\mathrm{B}_{1}$, respectively. (Right) Calculated electron energy loss spectra along the dashed lines indicated in the middle panel.

Returning to Fig. 1, we see that the lowest lying plasmon modes $\mathrm{S}_{1}$ are the symmetric and antisymmetric surface plasmons also predicted by the classical Drude model. The two sets of modes denoted $S_{2}$ and $S_{3}$ are also localized at

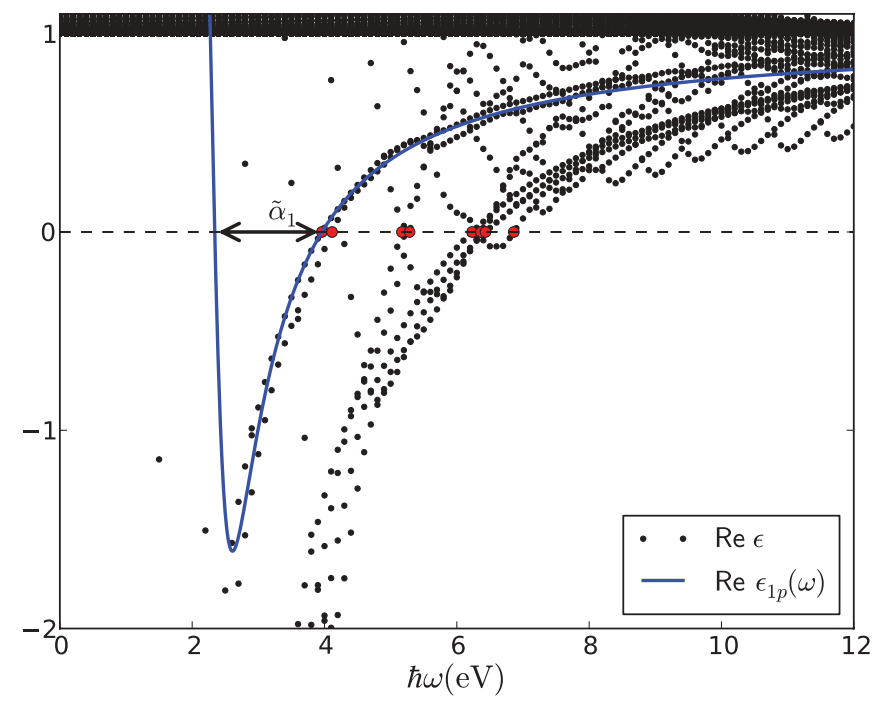

FIG. 2. (Color online) Real part of the eigenvalues of the microscopic dielectric function $\epsilon(\omega)$ for a ten layer $\mathrm{Na}$ film. The frequencies where an eigenvalue crosses the real axis from below (red dots) define the plasmon frequencies, and the corresponding eigenfunctions represent the plasmon modes shown by the red curves in the left panel of Fig. 1. The blue curve shows the real part of the single-pole model used to extract the strength of the plasmon. The distance between the zero points, $\tilde{\alpha}=\sqrt{\alpha^{2}-4 \gamma^{2}}$, increases with increasing strength of the mode. the surface, although they penetrate more into the bulk, and we therefore refer to them as subsurface modes. For a very thin slab of three layers, see Fig. 3, only the first subsurface mode, $S_{2}$, is supported. The subsurface character of the $S_{3}$ mode is perhaps more evident for the twenty layer film where it is more clearly separated from the bulk modes; see Fig. 4. Experimental loss spectra of simple metal surfaces have shown a small peak between the surface and bulk peaks which was assigned to a subsurface plasmon (in that work denoted multipole surface mode). ${ }^{23}$ Such a peak at intermediate energies was also observed in a previous RPA calculation for a jellium surface. ${ }^{24}$ However, the complete analysis of the plasmons modes presented here shows that more than one subsurface mode exists.

The bulk plasmons B occur at higher energies and resemble standing waves across the film. The fact that only a discrete

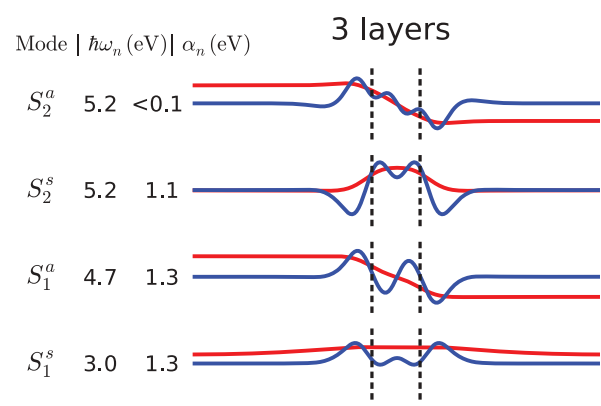

FIG. 3. (Color online) Spatial profile of the plasmon modes of a three layer $\mathrm{Na}$ film in the direction normal to the film for momentum transfer $q_{\|}=0.1 \AA^{-1}$. The red (blue) curves show the potential (density) associated with the plasmon excitation, and the mode type, energy, and strength $\alpha$ are shown to the left. 


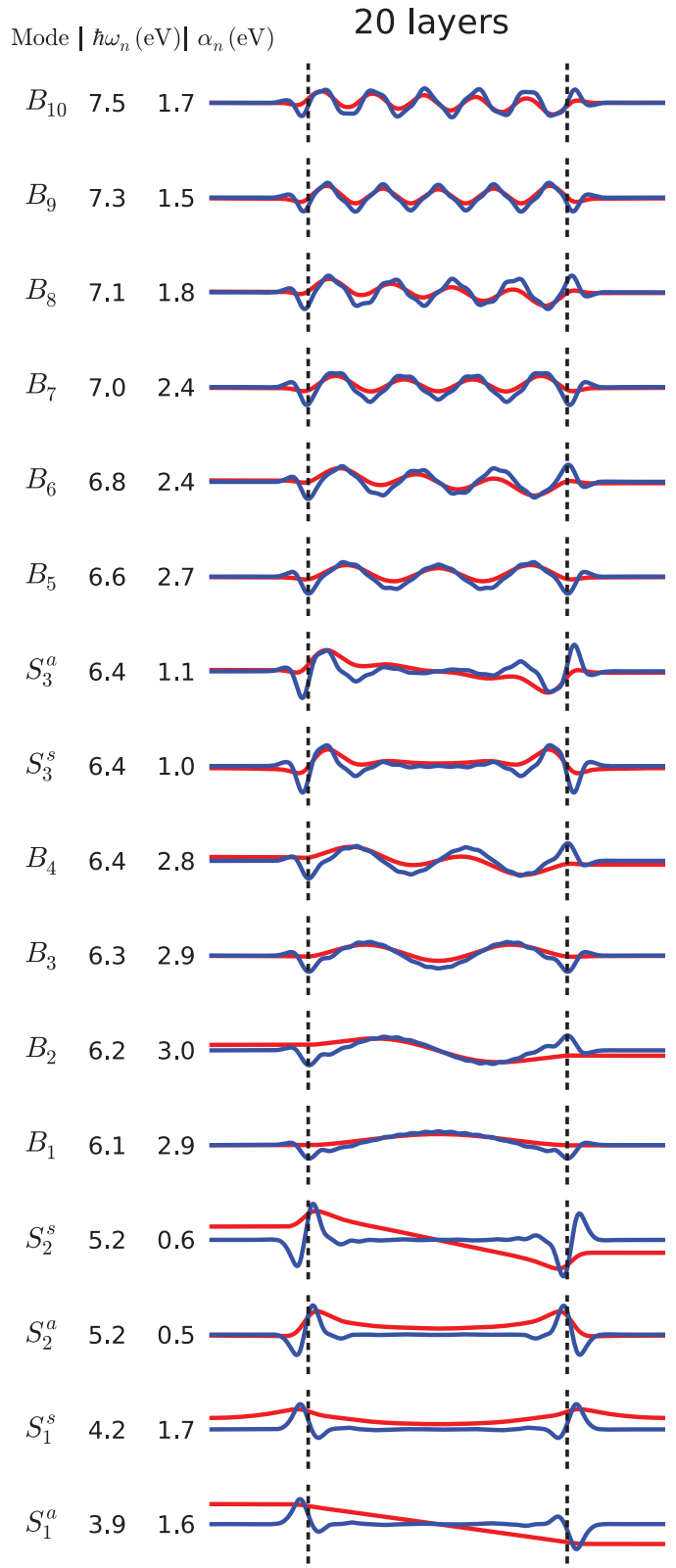

FIG. 4. (Color online) Spatial profile of the plasmon modes of a twenty layer $\mathrm{Na}$ film in the direction normal to the film for momentum transfer $q_{\|}=0.1 \AA^{-1}$. The red (blue) curves show the potential (density) associated with the plasmon excitation, and the mode type, energy, and strength $\alpha$ are shown to the left. In addition to the conventional surface modes $S_{1}$, the twenty layer slab supports two sets of subsurface plasmon modes $\left(S_{2}, S_{3}\right)$ with energies between the surface and bulk modes.

set of bulk modes are observed is clearly a result of the confinement of the electron gas which requires the density to vanish at the boundaries of the film. The reason only a finite number of modes are found is that the damping of the modes due to single-particle transitions increases for smaller oscillation periods, i.e., larger wave number. Consequently, the strength of the bulk plasmons decreases with increasing wave number until the point where the real part of $\epsilon$ does not cross the real axis. This is evident in Fig. 2 where a series of local minima in the eigenvalue spectrum can be seen at higher frequencies. For all the films, the highest lying bulk mode has a wave number around $0.5 \AA^{-1}$. This is very close to the threshold $q$ where $\operatorname{Re} \epsilon(q, \omega)$ becomes positive for all $\omega$ in bulk Na.

As already mentioned, the main mechanism of energy loss of fast electrons propagating through a material is via excitation of plasmons. In general, the energy dissipated to the electron system due to an applied potential of the form $\phi_{\mathrm{ext}}(r, t)=\phi_{\mathrm{ext}}(r) \exp (i \omega t)$ is

$$
P(\omega)=\iint \phi_{\mathrm{ext}}(r) \chi_{2}\left(r, r^{\prime}, \omega\right) \phi_{\mathrm{ext}}\left(r^{\prime}\right) d r d r^{\prime} .
$$

Here $\chi_{2}$ is the imaginary part of the density response function $\chi$. In the case of a fast electron, the external potential is simply that of a point charge moving at constant velocity. We have calculated the loss function for high energy electron beams directed along lines parallel to the film. The resulting EELS spectrum is seen in the middle panel of Fig. 1. It is clear that the loss spectrum is completely dominated by three types of excitations corresponding to the surface, subsurface, and bulk plasmon modes shown to the left. The intensity of the subsurface modes is rather weak in agreement with the low strength $\alpha$ of these modes.

Figure 5 shows all the energies of the symmetric and antisymmetric surface plasmons (subsurface plasmons not included) found for four different film thicknesses as a function of the in-plane wave vector $q_{\|}$(Ref. 25). The full curves are the classical Drude results for a two-dimensional (2D) metal film with the electron density of Na. For small $q_{\|}$the agreement with the classical result is striking for the symmetric mode. On the other hand, the quantum results for the antisymmetric mode are significantly red shifted compared to the Drude result with deviations up to $1 \mathrm{eV}$ for the thinnest film. For large $q_{\|}$the quantum plasmons show a $q_{\|}^{2}$ dispersion whereas the classical result approaches the asymptotic value $\omega_{p} / \sqrt{2}$. This

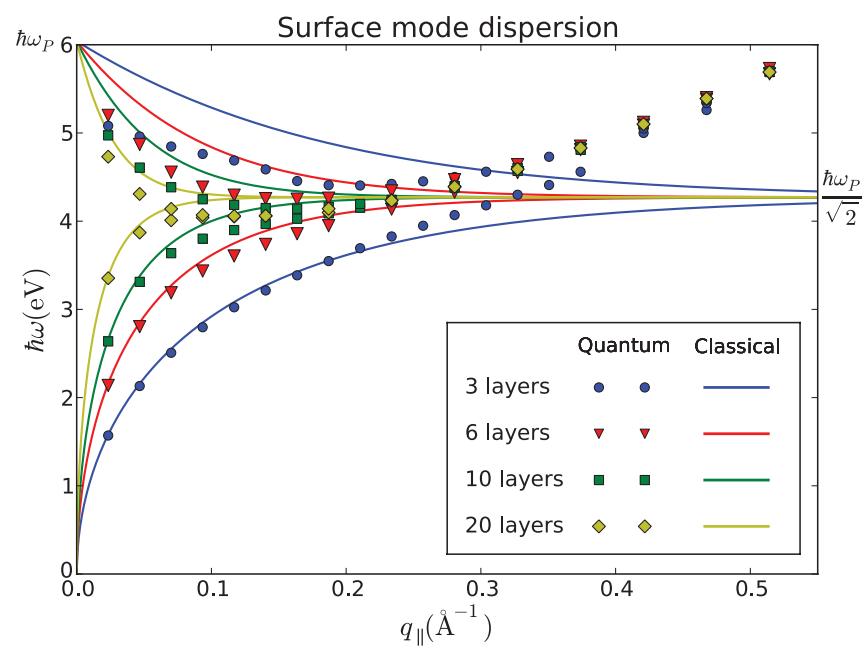

FIG. 5. (Color online) Dispersion of surface plasmon energies for different $\mathrm{Na}$ film thicknesses. The symbols represent the firstprinciples RPA results for the energy of the symmetric (lower branch) and antisymmetric (upper branch) surface plasmons as a function of the in-plane wave number $q_{\|}$. The full lines are the result of a classical Drude model. 


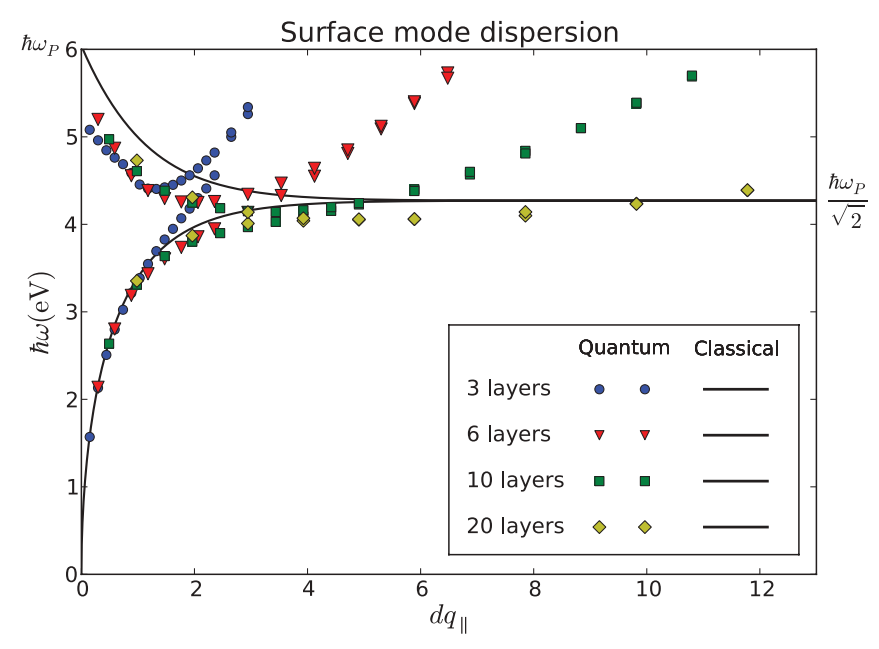

FIG. 6. (Color online) Like Fig. 5 but plotted as function of the dimensionless parameter $d q_{\|}$where $d$ is the thickness of the Na film.

failure of the classical model is due to the neglect of the spatial nonlocality of $\epsilon$, i.e., the $\left(r-r^{\prime}\right)$ dependence.

We note that when exchange-correlation effects are included through the adiabatic local density approximation (ALDA) kernel, a larger deviation from the classical model is observed. In particular the antisymmetric mode is shifted even further down for small $q_{\|}$. A similar behavior, i.e., a downshift of plasmon energies, is observed for simple metal bulk systems where the ALDA also predicts a negative dispersion for small $q$ (Ref. 2). The spatial form of the plasmon modes obtained with the ALDA is, however, identical to those obtained at the RPA level.

In Fig. 6 we show the same data as shown in Fig. 5 but with the plasmon energy plotted relative to the dimensionless parameter $d q_{\|}$, where $d$ is the film thickness. When plotted in this way, all the classical dispersions fall on the same universal curve. In the regime $d q_{\|}<2$, it is clear that the quantum results for the antisymmetric mode are not converged to the classical result even for the thickest slab.

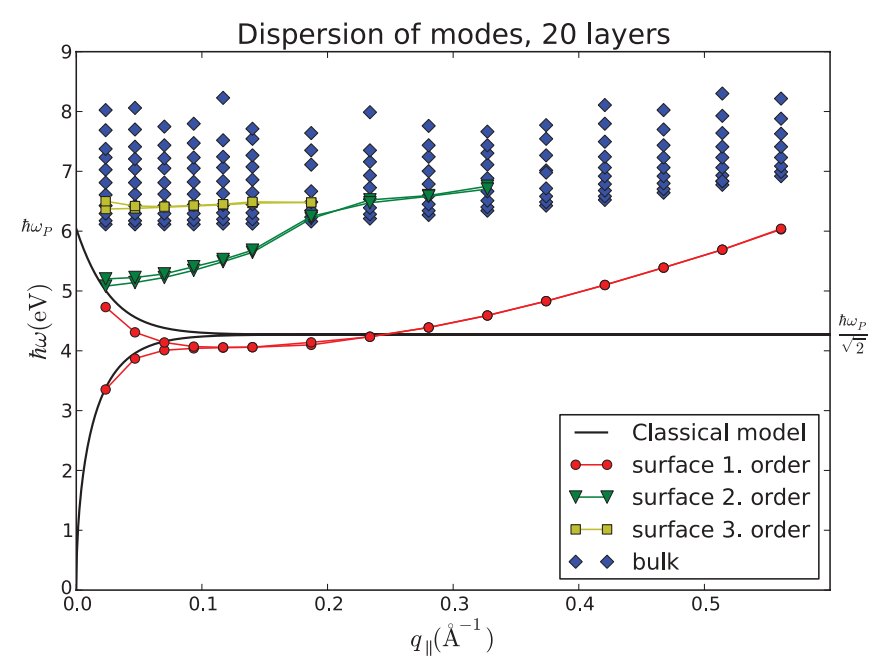

FIG. 7. (Color online) Dispersion of all the plasmon modes found for the twenty atomic layer $\mathrm{Na}$ film.
In Fig. 7 we present the dispersion of all plasmon modes (conventional surface, subsurface, and bulk modes) for the twenty layer film. The bulk modes show a weak $q_{\|}^{2}$ dispersion. The energy offset between the different bulk modes arises from the different wavelengths of the plasmons in the normal direction. The energy of the lowest bulk mode $\mathrm{B}_{1}$ in the $q_{\|}=0$ limit is $<0.1 \mathrm{eV}$ higher than the bulk value of $\hbar \omega_{p}$. This small deviation is due to the finite wavelength of the plasmon on the direction perpendicular to the film, and this indicates that quantum size effects have a very small influence on the bulk modes of the twenty layer film.

The first subsurface mode (green color) follows a $q_{\|}^{2}$ dispersion until around $0.2 \AA^{-1}$ where it enters the bulk mode energy range. From this point the dispersion of the subsurface mode is reduced and follows that of the bulk modes. The dispersion of the second subsurface mode (yellow color) is very similar to the bulk dispersion. The second subsurface mode is rather weak and for $q_{\|}>0.2 \AA^{-1}$ its eigenvalue $\epsilon_{n}(\omega)$ does not cross the real axis at any frequency. For both subsurface modes the splitting between the symmetric and antisymmetric modes is rather small. We ascribe this to the weaker strength of the electric field associated with the subsurface mode compared to the conventional surface mode: While the charge distribution $\rho_{n}$ associated with the latter has monopole character in the direction perpendicular to the film, the subsurface modes have dipole character; see Fig. 4.

\section{CONCLUSION}

We have demonstrated a method for calculating spatially resolved plasmon modes in nanostructured materials from first-principles. For the case of $2 \mathrm{D} \mathrm{Na}$ films ${ }^{19}$ of thicknesses 0.5 to $4 \mathrm{~nm}$, we found that the modes could be classified as either surface modes, subsurface modes, or bulk modes. In contrast to other studies, the direct computation of eigenmodes revealed that several subsurface modes can exist at the surface of simple metals. We found clear effects of quantum confinement on the surface plasmon energies. In particular, the antisymmetric surface mode of the thinner films was significantly red shifted compared to the classical Drude result. Finally, it was demonstrated how the different features in the calculated spatially resolved EELS spectrum of the metal films could be unambiguously ascribed to the excitation of specific plasmon modes. Apart from providing an intuitive and visual picture of the collective excitations of a nanostructure, the spatially resolved plasmon modes should be useful as a basis for the construction of simple models for the full nonlocal dielectric function.

\section{ACKNOWLEDGMENTS}

K.S.T. acknowledges support from the Danish Research Council's Sapere Aude Program. The Center for Nanostructured Graphene CNG is sponsored by the Danish National Research Foundation. Support from The Catalysis for Sustainable Energy (CASE) initiative and the Center of Nanostructuring for Efficient Energy Conversion (CNEEC) at Stanford University, an Energy Frontier Research Center funded by the US Department of Energy, Office of Science, Office of Basic Energy Sciences under Grant No. DE-SC0001060, is also acknowledged. 


\section{APPENDIX: SPECTRAL REPRESENTATION OF $\epsilon$}

Let $\left|\phi_{n}(\omega)\right\rangle$ denote the eigenfunctions of the dielectric function,

$$
\hat{\epsilon}(\omega)\left|\phi_{n}(\omega)\right\rangle=\epsilon_{n}(\omega)\left|\phi_{n}(\omega)\right\rangle .
$$

(We have suppressed the $r$ dependence for notational simplicity.) Since $\hat{\epsilon}(\omega)$ is a non-Hermitian operator, the eigenvalues are complex and the eigenfunctions are nonorthogonal. In this case the spectral representation takes the form

$$
\hat{\epsilon}(\omega)=\sum_{n} \epsilon_{n}(\omega)\left|\phi_{n}(\omega)\right\rangle\left\langle\rho_{n}(\omega)\right| .
$$

The set $\left|\rho_{n}(\omega)\right\rangle$ is the dual basis of $\left|\phi_{n}(\omega)\right\rangle$ and satisfies

$$
\left\langle\phi_{n}(\omega) \mid \rho_{n}(\omega)\right\rangle=\delta_{n m} .
$$

From the spectral representation it follows directly that

$$
\hat{\epsilon}(\omega)^{\dagger}\left|\rho_{n}(\omega)\right\rangle=\epsilon_{n}(\omega)^{*}\left|\rho_{n}(\omega)\right\rangle .
$$

In the following we show that the dielectric eigenfunction $\left|\phi_{n}(\omega)\right\rangle$ and its dual function $\left|\rho_{n}(\omega)\right\rangle$ constitute a potential- density pair, i.e.,

$$
\nabla^{2} \phi_{n}(r, \omega)=-4 \pi \rho_{n}(r, \omega) .
$$

Within the RPA, the dielectric function is related to the noninteracting polarization function $\chi_{0}\left(r, r^{\prime}, \omega\right)$ by

$$
\hat{\epsilon}(\omega)=\hat{1}-\hat{v} \hat{\chi}_{0}(\omega),
$$

where $\hat{v}=1 /\left|r-r^{\prime}\right|$ is the Coulomb interaction, $\hat{1}=\delta\left(r-r^{\prime}\right)$. Exploiting that, under time reversal symmetry $\chi_{0}\left(r, r^{\prime}\right)=$ $\chi_{0}\left(r^{\prime}, r\right)$, we have

$$
\hat{\epsilon}(\omega)^{\dagger}=\hat{1}-\hat{\chi}_{0}(\omega)^{*} \hat{v} .
$$

We now make the ansatz $\rho_{n}(\omega)=\hat{v}^{-1} \phi_{n}(\omega)^{*}$ and evaluate

$$
\begin{aligned}
\epsilon(\omega)^{\dagger} \hat{v}^{-1} \phi_{n}(\omega)^{*} & =v^{-1} \phi_{n}(\omega)^{*}-\hat{\chi}_{0}(\omega)^{*} \phi_{n}(\omega)^{*} \\
& =\hat{v}^{-1}\left[\hat{\epsilon}(\omega) \phi_{n}(\omega)\right]^{*} \\
& =\epsilon_{n}(\omega)^{*} \hat{v}^{-1} \phi_{n}^{*},
\end{aligned}
$$

which concludes the proof. *thygesen@fysik.dtu.dk

${ }^{1}$ D. Bohm and D. Pines, Phys. Rev. 92, 609 (1953).

${ }^{2}$ J. M. Pitarke, V. M. Silkin, E. V. Chulkov, and P. M. Echenique, Rep. Prog. Phys. 70, 1 (2007).

${ }^{3}$ K. A. Willets and R. P. Van Duyne, Annu. Rev. Phys. Chem. 58, 267 (2007).

${ }^{4}$ K. Kneipp, Y. Wang, H. Kneipp, L. T. Perelman, I. Itzkan, R. R. Dasari, and M. S. Feld, Phys. Rev. Lett. 78, 1667 (1997).

${ }^{5}$ E. M. Larsson, C. Langhammer, I. Zori, and B. Kasemo, Science 326, 1091 (2009).

${ }^{6}$ T. Hirakawa and P. V. Kamat, J. Am. Chem. Soc. 127, 3928 (2005).

${ }^{7}$ H. A. Atwater and A. Polman, Nat. Mater. 9, 205 (2010).

${ }^{8}$ F. J. Garcia de Abajo, Rev. Mod. Phys. 82, 209 (2010).

${ }^{9}$ B. S. Guiton, V. Iberi, S. Li, D. N. Leonard, C. M. Parish, P. G. Kotula, M. Varela, G. C. Schatz, S. J. Pennycook, and J. P. Camden, Nano Lett. 11, 3482 (2011); O. Nicoletti, M. Wubs, N. A. Mortensen, W. Sigle, P. A. van Aken, and P. A. Midgley, Opt. Express 19, 15371 (2011); W. Zhou, J. Lee, J. Nanda, S. T. Pantelides, S. J. Pennycook, and J. C. Idrobo, Nat. Nanotechnol. 7, 161 (2012); J. Nelayah, M. Kociak, O. Stèphan, F. J. Garcia de Abajo, M. Tencè, L. Henrard, D. Taverna, I. Pastoriza-Santos, L. M. Liz-Marzàn, and C. Colliex, Nat. Phys. 3, 348 (2007); A. L. Koh, A. I. Fernandez-Dominguez, D. W. McComb, S. A. Maier, and J. K. W. Yang, Nano Lett. 11, 1323 (2011); M. Bosman, V. J. Keast, M. Watanabe, A. I. Maaroof, and M. B. Cortie, Nanotechnol. 18, 165505 (2007); M. N'Gom, S. Li, G. Schatz, R. Erni, A. Agarwal, N. Kotov, and T. B. Norris, Phys. Rev. B 80, 113411 (2009); J. A. Scholl, A. L. Koh, and J. A. Dionne, Nature (London) 483, 421 (2012).

${ }^{10}$ C. David and F. J. Garcia de Abajo, J. Phys. Chem. C 115, 19470 (2011).

${ }^{11}$ S. Raza, G. Toscano, A. P. Jauho, M. Wubs, and N. A. Mortensen, Phys. Rev. B 84, 121412(R) (2011).

${ }^{12}$ T. Sokusin, A. Manjavacas, and F. J. Garcia de Abajo, ACS Nano 6, 1766 (2012).
${ }^{13}$ J. Yan, Z. Yuan, and S. W. Gao, Phys. Rev. Lett. 98, 216602 (2007).

${ }^{14}$ G. Onida, L. Reining, and A. Rubio, Rev. Mod. Phys. 74, 601 (2002).

${ }^{15}$ C. Kramberger, R. Hambach, C. Giorgetti, M. H. Rümmeli, M. Knupfer, J. Fink, B. Büchner, L. Reining, E. Einarsson, S. Maruyama, F. Sottile, K. Hannewald, V. Olevano, A. G. Marinopoulos, and T. Pichler, Phys. Rev. Lett. 100, 196803 (2008).

${ }^{16}$ A. Marini, R. Del Sole, and G. Onida, Phys. Rev. B 66, 115101 (2002).

${ }^{17}$ J. Yan, K. W. Jacobsen, and K. S. Thygesen, Phys. Rev. B 84, 235430 (2011)

${ }^{18}$ We note that exact solutions may be obtained for complex frequencies. However, this requires an analytical continuation of $\epsilon$ into the complex plane and will not be pursued in the present work.

${ }^{19}$ In the case of Ag films (results will be published elsewhere), we have found that the coupling to interband transitions can shift the peak of $\operatorname{Im} \epsilon_{n}(\omega)$ away from the point where $\operatorname{Re} \epsilon_{n}(\omega)=0$. Importantly, however, the corresponding eigenfunction $\phi_{n}(r, \omega)$ does not change significantly when $\omega$ is varied between the frequencies given by the two criteria, and thus the spatial form of the plasmon remains well defined.

${ }^{20}$ J. Yan, J. J. Mortensen, K. W. Jacobsen, and K. S. Thygesen, Phys. Rev. B 83, 245122 (2011).

${ }^{21}$ J. Enkovaara et al., J. Phys.: Condens. Matter 22, 253202 (2010).

${ }^{22}$ S. R. Bahn and K. W. Jacobsen, Comput. Sci. Eng. 4, 56 (2002).

${ }^{23}$ K.-D. Tsuei, E. W. Plummer, A. Liebsch, E. Pehlke, K. Kempa, and P. Bakshi, Surf. Sci. 247, 302 (1991).

${ }^{24}$ W. L. Schaich and J. F. Dobson, Phys. Rev. B 49, 14700 (1994).

${ }^{25}$ To obtain the dispersion of the surface plasmons (Fig. 4) of an isolated film, the calculated plasmon energies were extrapolated to the limit of infinite distance between the repeated films using a classical model for the plasmon energies in an infinite array of films. The model is a straightforward generalization of the results in Ref. 26.

${ }^{26}$ R. A. Ferrell, Phys. Rev. 111, 1214 (1958). 